\title{
EFEITO DO TREINO NEUROMUSCULAR NA ROTAÇÃO DO JOELHO DURANTE A ATERRISSAGEM EM MULHERES
}

\author{
NEUROMUSCULAR TRAINING EFFECT ON KNEE ROTATION DURING DROP LANDING IN WOMEN
}

EFECTO DEL ENTRENAMIENTO NEUROMUSCULAR EN LA ROTACIÓN DE LA RODILLA DURANTE EL ATERRIZAJE EN LAS MUJERES

Narayana Vianna Spanó ${ }^{1,2}$

(Educadora Física)

Fábio Pamplona Mariano 1,2

(Fisioterapeuta)

Vitor Luiz de Andrade ${ }^{1,2}$

(Educador Físico)

Bruno Luiz de Souza Bedo 1,2

(Educador Físico)

Luiz Henrique Palucci Vieira ${ }^{1,2}$

(Educador Físico)

Paulo Roberto Pereira Santiago 1,2

(Educador Físico)

1. Universidade de São Paulo, Faculdade de Medicina de Ribeirão Preto, Programa de Pós-Graduação em Reabilitação e Desempenho Funcional, Ribeirão Preto, Brasil. 2. Universidade de São Paulo, Escola de Educação Física e Esporte de Ribeirão Preto, Laboratório de Biomecânica e Controle Motor, Ribeirão Preto, Brasil.

\section{Correspondência:}

Paulo Roberto Pereira Santiago. Universidade de São Paulo, Escola de Educação Física e Esporte de Ribeirão Preto. Av. Bandeirantes, n. 3900. Monte Alegre,

Ribeirão Preto, SP, Brasil. 14040-907. paulosantiago@usp.br

\section{RESUMO}

Introdução: O grande índice de lesões do ligamento cruzado anterior sem envolver contato em mulheres é motivo de curiosidade entre os cientistas, pois avaliações de aterrissagem podem sugerir a predisposição ao risco dessa lesão. Neste sentido, vários protocolos de treinamento foram utilizados como intervenção e obtiveram resultados diversificados na alteração desse fator de risco. Objetivo: O objetivo deste projeto foi avaliar as possíveis alterações de rotação do joelho na aterrissagem unipodal após a intervenção de um programa de treinamento neuromuscular. Métodos: Participaram do estudo 18 mulheres com idade entre $18 \mathrm{e}$ 51 anos, que foram distribuídas em dois grupos: grupo de treinamento neuromuscular de oito semanas (GTN) $(n=11)$ e grupo controle $(G C)(n=7)$. Além disso, não apresentavam lesão musculoesquelética ou dores nos membros inferiores. Cada participante realizou cinco aterrissagens unipodais válidas de uma plataforma de $40 \mathrm{~cm}$ de altura. O processo de captura de movimento foi realizado com 12 câmeras infravermelho do sistema OptiTrack ${ }^{\text {TM }}$ para obter as coordenadas tridimensionais de marcadores fixados nos pontos anatômicos de interesse. Os sistemas de coordenadas locais da coxa e da perna foram definidos por meio dos respectivos marcadores fixados nos pontos anatômicos. Dessa forma, foram calculados os ângulos de rotação do joelho nos planos sagital, frontal e transverso através das sequências dos ângulos de Euler e as velocidades angulares através das formulações dos quatérnions. Resultados: Os resultados mostraram que houve um aumento na velocidade de rotação do joelho após o treinamento neuromuscular. Conclusão: Conclui-se que o treinamento neuromuscular resultou em maior velocidade de rotação no joelho nos 40 milissegundos que sucedem a aterrissagem unipodal.

Descritores: fenômenos biomecânicos; joelho; aptidão física.

\section{ABSTRACT}

Introduction: The high rate of injuries of the anterior cruciate ligament (ACL) without involving contact in women is a source of curiosity among scientists, because drop landing assessments may suggest a predisposition to the risk of this injury. In these sense, several training protocols have been used as an intervention and achieved mixed results in the change of this risk factor. Objective: The aim of this project was to evaluate the possible changes of rotation knee in single-leg drop landing after the intervention of a neuromuscular training program. Methods: The study included 18 women aged between 18 and 51 years, who were divided into two groups: neuromuscular training group (NTG) ( $n=11)$ and control group (CG) (n=7). In addition, they did not have musculoskeletal injury or pain in the lower limbs. Each participant completed five valid single-leg drop landings from a platform of $40 \mathrm{~cm}$. The motion capture process was carried out with 12 infrared cameras of the OptiTrack ${ }^{\mathrm{TM}}$ system to obtain three-dimensional coordinates of markers fixed in the anatomical points of interest. The local coordinate systems of the thigh and leg were defined by means of the respective markers set in the anatomical points. Thus, the knee rotation angles in the sagittal, frontal, and transverse planes were calculated through the sequence of Euler angles and the angular velocities were calculated using the formulations of quaternions. Results: The results showed an increase in the knee rotation speed after the neuromuscular training. Conclusion: We conclude that neuromuscular training resulted in faster rotation at the knee in the 40 milliseconds succeeding the single-leg landing.

Keywords: biomechanical phenomena; knee; physical fitness.

\section{RESUMEN}

Introducción: El gran número de lesiones del ligamento cruzado anterior sin que implique un contacto en las mujeres es motivo de curiosidad en tre los científicos porque las evaluaciones de aterrizaje pueden sugerir predisposición al riesgo de esta lesión. En este sentido, varios protocolos de entrenamiento se han utilizado como intervención y lograron resultados mixtos en el cambio de este factor de riesgo. Objetivo: El objetivo de este proyecto fue evaluar los posibles cambios de rotación de la rodilla en el aterrizaje unipodal después de la intervención de un programa de entrenamiento neuromuscular. Métodos: El estudio incluyó a 18 mujeres de edades comprendidas entre 18 y 51 años, que fueron divididas en dos grupos: grupo de entrenamiento neuromuscular de ocho semanas (GEN) $(n=11)$ y grupo control (GC) $(n=7)$. Además, no tenían lesión musculoesquelética o dolor en las extremidades inferiores. 
Cada participante realizó cinco aterrizajes unipodales válidos desde una plataforma de $40 \mathrm{~cm}$. El proceso de captura de movimiento se llevó a cabo con 12 cámaras infrarrojas del sistema OptiTrack ${ }^{\mathrm{TM}}$ para obtener las coordenadas tridimensionales de los marcadores fijados a los puntos anatómicos de interés. Los sistemas de coordenadas locales del muslo y la pierna se definieron por medio de los respectivos marcadores establecidos en los puntos anatómicos. De esa manera, fueron calculados los ángulos de rotación de la rodilla en los planos sagital, frontal y transversal utilizando las secuencias de los ángulos de Euler, y las velocidades angulares utilizando las formulaciones de cuaterniones. Resultados: Los resultados mostraron un aumento en la velocidad de rotación de la rodilla después del entrenamiento neuromuscular. Conclusión: Se concluye que el entrenamiento neuromuscular resultó en mayor velocidad de rotación en la rodilla en los 40 milisegundos posteriores al aterrizaje unipodal.

Descriptores: fenómenos biomecánicos; rodilla; aptitud física.

\section{INTRODUÇÃO}

Diversos trabalhos demonstram a importância da análise cinemática para aprimorar a execução de movimentos, assim como avaliar padrões de mecanismos de lesão e até prevenir lesões em gestos esportivos ${ }^{1-3}$.

Diferenças no padrão de movimento entre os sexos são investigados pela incidência desproporcional de lesões do ligamento cruzado anterior (LCA) e síndrome femoropatelar desenvolvidas em mulheres ${ }^{4}$. Estas diferenças podem ser justificadas por fatores anatômicos ${ }^{5}$, biomecânicos ${ }^{1,6}$, neuromusculares $s^{7,8}$ e hormonais ${ }^{9,10}$. Dentre os presentes fatores modificáveis como a biomecânica e o neuromuscular são importante pontos para desenvolvimento de programas de prevenção de lesões, que poderiam ser alterados com treinamentos adequados ${ }^{3}$.

O controle neuromuscular é importante para a execução dos movimentos durante o esporte, sua alteração ou diminuição pode resultar em movimentos desnecessários e excessivos das articulações dos membros inferiores levando em muitos casos á diversas lesões, como a ruptura do LCA ${ }^{11}$. Além disso, o treinamento neuromuscular possui como característica básica melhorar a informação de respostas motoras involuntárias, através dos estímulos aferentes e dos mecanismos centrais responsáveis pelo controle dinâmico ${ }^{12,13}$.

Durante aterrissagem unipodal, no âmbito da biomecânica, as muIheres apresentam maior adução e rotação medial do fêmur em relação à tíbia, as quais produzem maior amplitude de valgo quando comparado aos homens ${ }^{14}$, padrão que predispõe a lesões. Essa maior incidência pode estar associada ao fato das mulheres apresentarem menor ângulo de flexão do joelho ${ }^{15}$. Uma estratégia para promover condições ideais e prevenir possíveis lesões músculo esquelética no joelho é a distribuição regular da componente vertical de reação do solo, ou seja, controlar o deslocamento angular máximo das rotações do joelho durante flexão do joelho. Dessa forma a acomodação da articulação do joelho durante a aterrissagem não sofrerá alterações biomecânicas, e assim pode apresentar melhores condições para atenuar a força gerada nessa tarefa ${ }^{16}$.

Com isso, o objetivo do estudo foi analisar as alterações na cinemática de rotação do joelho, através doa ângulos de Euler e das velocidades angulares pelos quatérnions, na aterrissagem unipodal após um programa de treinamento neuromuscular, com a hipótese que o treino neuromuscular poderia alterar o padrão de movimento de rotação do joelho das mulheres através do aprendizado motor e consequente melhorar o desempenho na tarefa de aterrissagem unipodal.

\section{MATERIAIS E MÉTODOS}

Inicialmente foram recrutadas 35 voluntárias do sexo feminino, saudáveis entre 18 e 51 anos de idade, com dominância pedal direita. Como critério de exclusão as voluntárias não poderiam apresentar histórico de lesões musculoesqueléticas ou ter passado por processos cirúrgicos nos membros inferiores. Assim como não poderiam sofrer de dores articulares nos membros inferiores durante atividade diárias ou no momento das avaliações.

As participantes foram divididas em dois grupos: grupo treinamento neuromuscular (GTN) com 11 voluntárias e grupo controle (GC) com sete voluntárias (Figura 1).

No momento da inclusão na pesquisa todas as participantes assinaram o Termo de Consentimento Livre e Esclarecido de acordo com a resolução 196/96 do Conselho Nacional de Saúde e aprovado pelo Comitê de Ética em Pesquisa da Instituição e aprovado pelo Comitê de Ética em Pesquisa da Faculdade de Medicina de Ribeirão Preto sob o protocolo (06204712.5.0000.5440).

Primeiramente as voluntárias preencheram um questionário para obter informações sobre estilo de vida como: atividades que praticam atualmente, históricos das atividades praticadas, além de dados antropométricos.

Após a aplicação do questionário foram colocados doze marcadores retro refletivos com dez milímetros de diâmetro fixados nas seguintes proeminências ósseas do quadril e membro inferior direito: primeira vértebra sacral, espinha ilíaca antero-superior direita e esquerda, trocânter maior do fêmur, epicôndilo lateral do fêmur, epicôndilo medial do fêmur, cabeça da fíbula, maléolo lateral, maléolo medial, tuberosidade do calcâneo, cabeça do quinto metatarso e cabeça do primeiro metatarso ${ }^{17}$.

Com os marcadores posicionados as voluntárias foram instruídas a realizarem cinco aterrissagens unipodais validas com o membro inferior direito. O posicionamento inicial foi em cima da plataforma de 40 centímetros de altura em posição corporal ortostática com os pés em paralelo, com mãos posicionadas sobre a crista ilíaca, e foram orientadas a realizar um passo

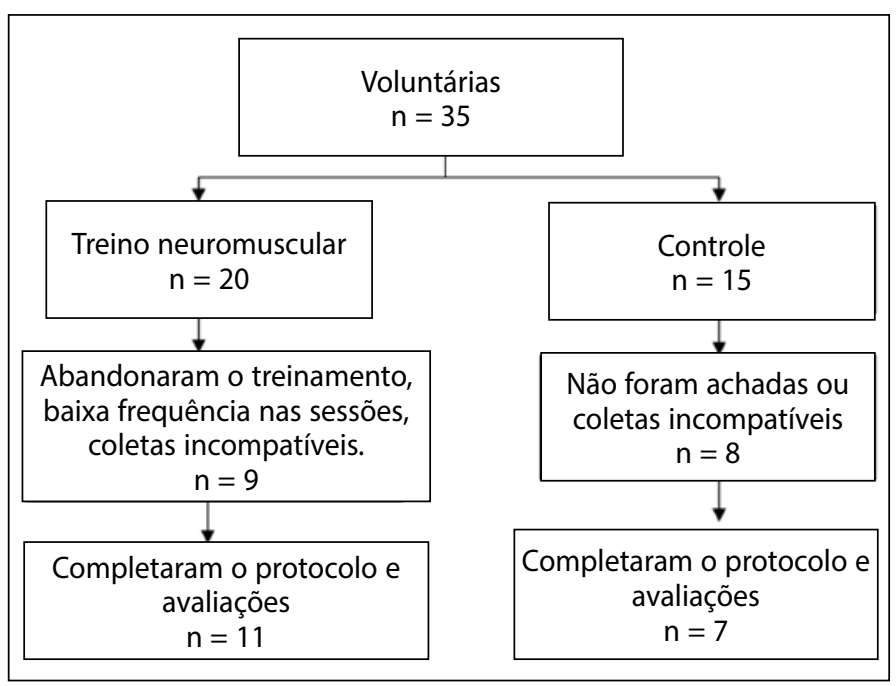

Figura 1. Fluxograma das participantes do Grupo Controle e Treino Neuromuscular convidadas para participar do estudo. 
para fora da plataforma, deslocando o corpo a frente, sem saltar ou pular, porém executar o movimento de aterrissarem final sob apoio unipodal.

As avaliações das aterrissagens unipodais foram realizadas antes e após o período de treinamento tanto no GTN quanto no GC.

A aquisição dos dados cinemáticos foi através do sistema OptiTrack ${ }^{T M}$, o qual era constituído de doze câmeras infravermelhas, sincronizadas a $250 \mathrm{~Hz}$ e conectadas a um computador para a aquisição dos dados cinemáticos em 3D.

O protocolo de treinamento neuromuscular (TN) foi constituído por exercícios de equilíbrio estático e dinâmico, pliométricos, de coordenação motora e agilidade ${ }^{18}$.

O GTN realizou em média seis semanas de treinamento, com frequência de três vezes por semana, com duração de 40 minutos cada sessão, totalizando de dez a dezoito sessões de treino. Na primeira semana de treinamento foram realizadas sessões com intuito de adaptação e aprendizagem da execução dos exercícios presentes no TN. Todos os exercícios foram cronometrados durante a execução com objetivo de quantificar o tempo gasto para executar cada exercício e foi utilizado a percepção subjetiva de esforço pela escala de Borg para obter dados do esforço realizado em cada exercício e assim garantir o nível de dificuldade durante as sessões de treinamento.

\section{Análise de dados}

As análises dos dados foram realizadas através do processamento de rotinas criadas no software MatLab ${ }^{\circledR}$ (Matchworks Inc.,Natick,MA,USA).

No sentido de padronizar o movimento de análise, o mesmo período de tempo foi considerado em todas as aterrissagens, sendo que o contato inicial (Cl) do pé com o solo foi definido como o evento comum para todas as aterrissagens. A identificação do $\mathrm{Cl}$ do pé foi realizada por meio da coordenada tridimensional que corresponde a um componente vertical do marcador fixado na cabeça do primeiro metatarso. Desta forma a desaceleração na componente vertical pode ser utilizada para detectar o instante de $\mathrm{Cl}$ do pé com o solo. A partir desse ponto o intervalo de análise foi estipulado entre 300 ms antes e após o contato inicial.

\section{Cálculo dos ângulos de Euler e dos quatérnions}

Antes do cálculo dos ângulos de Euler as coordenadas espaciais de cada marcador foram filtrados por um filtro digital passa-baixa do Butterworth de quarta ordem com frequência de corte de oito hertz.

Para o cálculo dos ângulos de Euler e do quatérnion unitário que representou a rotação ocorrida na articulação do joelho foram construídos os sistemas de referência local da coxa e da perna. Os sistemas de referências locais foram definidos pelos versores i, j e k construídos através das coordenadas tridimensionais de cada marcador, para o sistema coxa foram utilizados os marcadores do trocânter maior do fêmur, epicôndilo lateral do fêmur, epicôndilo medial do fêmur, já o sistema perna foi definido com os marcadores da cabeça da fíbula, maléolo lateral, maléolo medial, a partir esses versores seguiram orientações definidas por Grood e Suntay ${ }^{19}$.

Uma vez definidos os sistemas de referências locais foram possíveis os cálculos para a obtenção dos ângulos de Euler a partir das matrizes de rotação que representam os movimentos rotacionais ocorridos na articulação do joelho ${ }^{20}$. Para isso foi utilizado a função do software Matlab dcm2angle.m com o parâmetro de entrada XYZ, para a sequência de rotação. Assim, o cálculo das rotações ocorridas foi padronizado de acordo com a rotação ocorrida do segmento distal (perna) em relação ao segmento proximal (coxa). $O$ eixo $X$ representa a flexão e extensão do joelho, o eixo Y a adução e abdução e o eixo Z as rotações interna e externa do joelho.

Para o cálculo da velocidade angular optou-se pelo formalismo matemático dos quatérnions, utilizando as mesmas matrizes de rotação utilizadas para o cálculo dos ângulos de Euler ${ }^{21}$.

\section{Análise Estatística}

Os ângulos de Euler e as velocidades angulares via quatérnions foram comparados nos momentos pré e pós por meio de teste t pareado, nas condições intragrupos (Pré-GTN x Pós-GTN e Pré-GC x Pós-GC). Para as comparações intergrupos utilizou teste t para amostras independentes (Pré-GTN x Pré-GC, Pós-GTN x Pós-GC). Em todas as situações foi adotado nível de significância 5\% ( $p \leq 0,05)$.

\section{RESULTADOS}

As características antropométricas nos momentos pré e pós estão apresentadas em valores de média e desvio padrão na Tabela 1.

O teste t pareado não revelou diferenças significativas nas variáveis antropométricas para os dois momentos da avaliação do estudo.

Para todas as análises de rotação expressa pelos ângulos de Euler optou-se pelo tempo de 40 ms após o contato inicial, pois esse é o momento mais suscetível para lesão do joelho em tarefas de aterrissagem e troca de direção no sexo feminino ${ }^{22}$

Nas comparações dos ângulos de flexão do joelho o teste t pareado não revelou diferença entre os momentos pré e pós no tempo de 40 ms após o $\mathrm{Cl}$ para o GTN ( $p=0,785)$. Contudo, foi verificada diferença entre o momento pré e pós para o GC ( $p=0,019)$ (Figura 2).

O teste t para amostras independentes para flexão do joelho no tempo de 40 ms, não revelou diferenças entre GTN e GC nos momentos pré $(p=0,399)$ e pós $(p=0,627)$.

O teste t pareado para abdução do joelho no tempo de $40 \mathrm{~ms}$ após $\mathrm{Cl}$, não revelou diferença entre o momento pré e pós para o GTN $(p=0,960)$ e $G C(p=0,547)$ (Figura 3).

O teste t para amostras independentes para abdução do joelho no tempo de 40 ms, não revelou diferenças entre GTN e GC nos momentos pré $(p=0,967)$ e pós $(p=0,519)$.

O teste t pareado não revelou diferença para rotação interna do joelho no tempo de 40 ms após o $\mathrm{Cl}$ entre os momentos pré e pós para o GTN ( $p=0,446)$ e GC ( $p=0,511)$ (Figura 4).

$O$ teste $t$ para amostras independentes para rotação interna do joelho no tempo de 40 ms, não revelou diferenças entre GTN e GC nos momentos pré $(p=0,161)$ e pós $(p=0,090)$.

Tabela 1. Dados antropométricos das participantes do estudo representados em média e desvio padrão.

\begin{tabular}{c|c|c|c}
\hline & Estatura $(\mathbf{c m})$ & Massa $(\mathbf{k g})$ & Idade (anos) \\
\hline Pré-GTN & $162,6 \pm 5,3$ & $65,9 \pm 6,3$ & $27,1 \pm 13,2$ \\
\hline Pós-GTN & $162,5 \pm 5,2$ & $64,9 \pm 5,2$ & $27,1 \pm 13,2$ \\
\hline Pré-GC & $160,3 \pm 4,8$ & $61,2 \pm 6,9$ & $31,0 \pm 12,1$ \\
\hline Pós-GC & $160,3 \pm 4,8$ & $60,1 \pm 5,8$ & $31,0 \pm 12,1$ \\
\hline
\end{tabular}

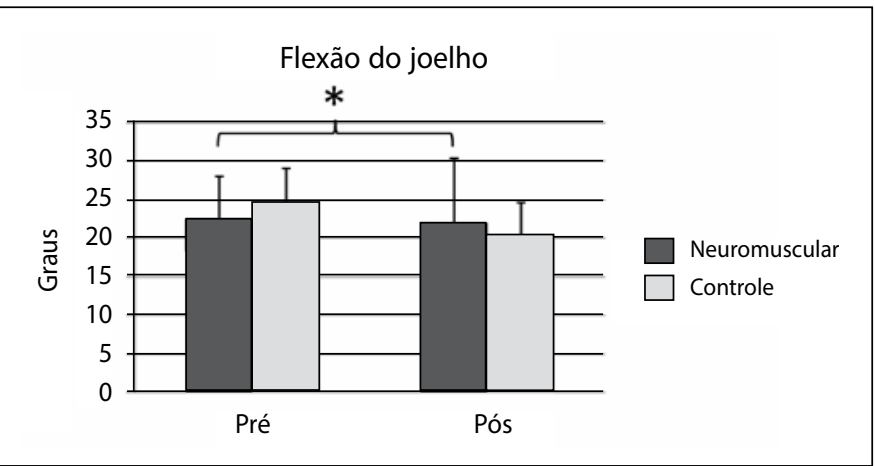

Figura 2. Ângulo de flexão do joelho nos momentos pré e pós dos grupos controle e neuromuscular. (*) Diferença significativa entre os momentos pré e pós do grupo controle. 
O teste t pareado para a velocidade máxima de rotação expressa pelo quatérnions, comparando os momentos pré e pós, revelou diferença para GTN ( $p=0,043)$, mas não revelou diferença para GC $(p=0,282)$ (Figura 5).

O teste t para amostras independentes não revelou diferenças na velocidade máxima de rotação expressa pelo quatérnions entre os GTN e GC nos momentos pré ( $p=0,453)$ e pós $(p=0,293)$.

Contudo, o teste t para amostras independentes mostrou diferença para a velocidade máxima de rotação no delta de variação no momento pré para pós entre GTN e GC ( $p=0,027)$. Nesse caso, verifica-se que GTN teve um aumento na velocidade de rotação enquanto o GC apresentou uma redução significativa nessa variável.

Não foi verificada diferença no teste t pareado para o tempo associado à máxima velocidade de rotação após o $\mathrm{Cl}$, tanto no momento pré e pós do GTN ( $p=0,549)$ e GC $(p=0,446)$. Assim como, não foram verificadas diferenças no teste t para amostras independentes no momento pré entre GTN e GC ( $p=0,943)$ e no momento pós entre GTN e $G C(p=0,231)$ (Figura 6).

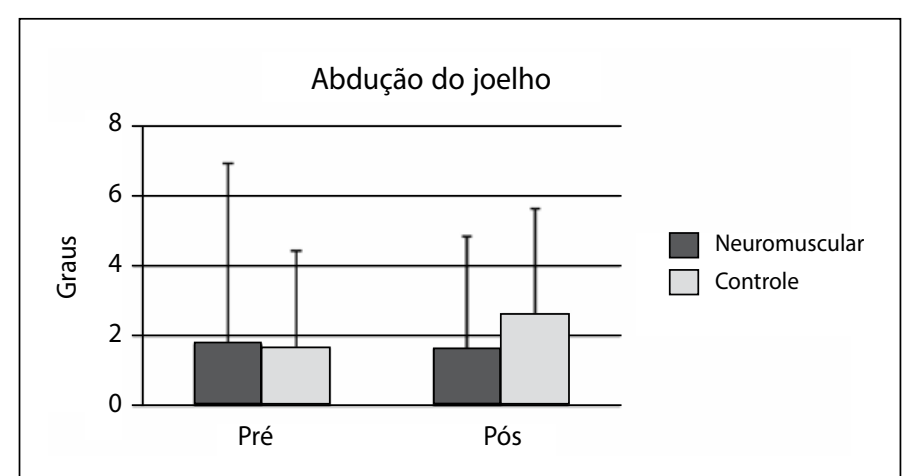

Figura 3. Ângulo de abdução do joelho pré e pós para os grupos controle e neuromuscular.

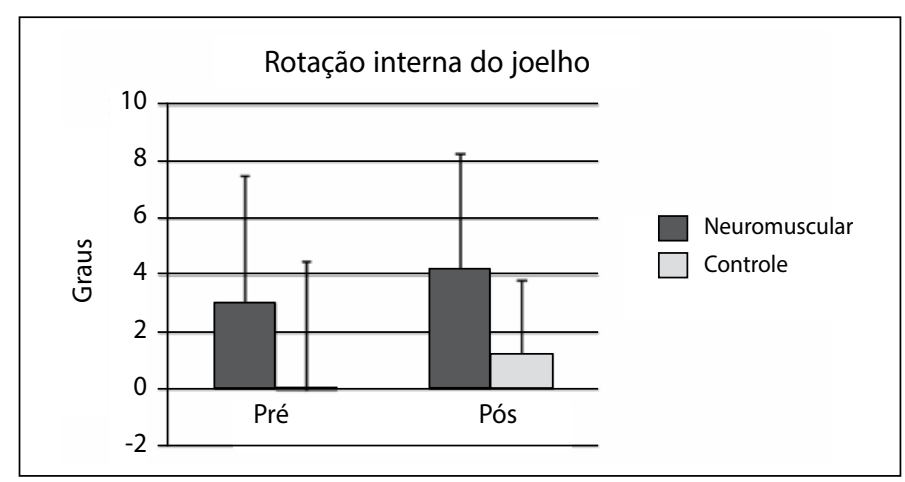

Figura 4. Ângulo de rotação interna do joelho nos momentos pré e pós dos grupos controle e neuromuscular.

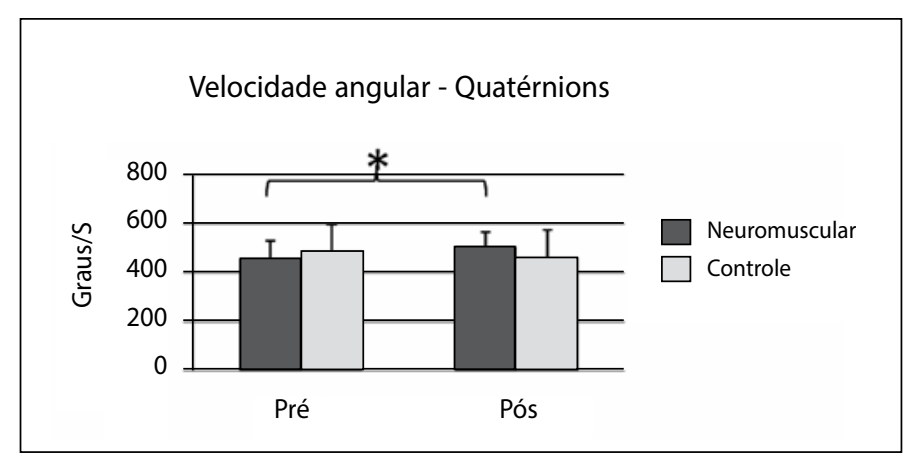

Figura 5. Velocidade angular de rotação do joelho expresso pelo quatérnion unitário em graus por segundo. (*) Diferença significativa entre os momentos pré e pós para o grupo reino neuromuscular.
Tempo de máxima velocidade angular Quatérnions

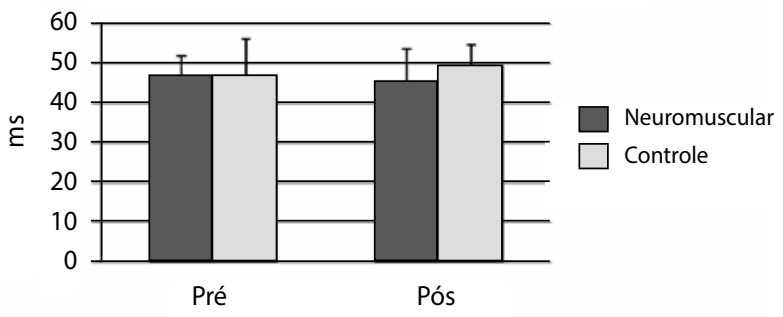

Figura 6. Tempo em milissegundos correspondente a máxima velocidade angular de rotado joelho expressa pelo quatérnion unitário.

\section{DISCUSSÃO}

Durante a tarefa de aterrissagem no tempo de 40 ms, o GTN não apresentou diferenças pré e pós-treinamento no ângulo de flexão de joelho, mas para o grupo controle o ângulo de flexão do joelho diminuiu de pré para pós no período de 14 semanas. $O$ maior ângulo de flexão do joelho, quadril e tronco durante a aterrissagem estão associados à redução do risco de lesão do LCA ${ }^{23,24}$. Pesquisas que compararam grupos de participantes que apresentam padrões com menor flexão de joelhos com relação aos que possuem padrão com maior flexão de joelhos durante aterrissagem, concluíram que o grupo que exibia menor flexão de joelhos aumentou ângulo do valgo da articulação do joelho, aumentou momento abdutor da articulação do joelho, diminuiu a energia de absorção das articulações do joelho e quadril e aumentou estímulo eletromiográfico do vasto lateral em relação ao grupo com padrão de maior flexão de joelhos. Este padrão biomecânico exibido pelo grupo de menor flexão de joelhos com limitação de movimento no plano sagital pode levar a um maior risco de lesão de LCA ${ }^{25}$.

A velocidade angular do grupo neuromuscular apresentou diferença entre pré e pós intervenção, o que corrobora com resultados encontrados na literatura ${ }^{24,26-28}$. Os resultados do estudo Yeow et al. ${ }^{24}$ vão ao encontro dos resultados apresentados neste estudo, em que o grupo GTN aumentou velocidade angular do período pré para pós treinamento e o grupo controle apresentou diminuição o ângulo de flexão do joelho em relação ao mesmo período.

Na variável do ângulo de abdução do joelho não foi observado alterações pré e pós GTN semelhantes aos resultados encontrados na literatura ${ }^{29}$. Neste estudo foram a amostra foi composta por 30 atletas de basquete que realizaram treinamento neuromuscular por seis semanas e não apresentaram alteração na variável do momento de valgo dinâmico durante a tarefa do Drop jump, mas ocorreram alterações apenas na tarefa Stop jump.

Existem alguns indícios na literatura ${ }^{30}$ que o treino neuromuscular apresente alterações na cinemática de tarefas específicas, como é o caso do single leg drop-landing. Todavia, o presente estudo não apresentou alterações nos ângulos de abdução e rotação externa do joelho, que são os responsáveis pelo fenômeno valgo dinâmico.

Uma hipótese para a não alteração dessas variáveis pode estar no fato do treino neuromuscular aplicado neste estudo ter enfatizado agilidade através dos exercícios de saltos, e a ênfase na velocidade de execução através das avaliações que foram realizadas por tempo de execução. Estas variáveis podem ter influenciado em uma maior velocidade angular de rotação do joelho, verificado através do quatérnion unitário. Isso é de extrema relevância do ponto de vista da preservação das estruturas moles (músculos, tendões e ligamentos), pois é possível que as voluntárias que realizaram o treino neuromuscular tenham adquirido uma capacidade de produzir o movimento de rotação da articulação do joelho após o contato com maior velocidade, e assim, podem acomodar com maior 
eficiência as estruturas dos membros inferiores com intuito de reduzir as cargas impostas na articulação do joelho.

Na variável tempo pico da máxima velocidade de rotação após o Cl também foi verificado que o GTN reduziu o tempo na comparação do momento pré e pós-treinamento e no delta variação demonstrou que comparado ao GC o GTN reduziu o tempo para atingir o pico de máxima velocidade de rotação do joelho, ao passo que o GC aumentou este tempo no momento pré e pós quatorzes semanas. O GTN aumentou a agilidade e melhorou tempo para reorganização das estruturas corporais com eficiência durante a tarefa de aterrissagem.

\section{CONCLUSÃO}

Com base nos resultados obtidos no presente estudo pode-se concluir que o protocolo de treinamento neuromuscular idealizado resultou em uma maior velocidade de rotação no joelho nos 40 milissegundos que sucedem a aterrissagem unipodal. Considera-se que esse fator permite uma melhora na acomodação do joelho na aterrissagem unipodal, que confirma a eficácia do protocolo de treinamento neuromuscular como estratégia de prevenção de lesões no joelho.

\section{AGRADECIMENTOS}

Fundação de Amparo à Pesquisa do Estado de São Paulo - FAPESP, processos No [2010/20538-7] e [2011/14811-5]. Os autores agradecem a Eduardo Bergonzoni Junqueira e Andréia Abud da Silva Costa, que ajudaram no treinamento e coleta de dados.

Todos os autores declararam não haver qualquer potencial conflito de interesses referente a este artigo.

CONTRIBUIÇÕES DOS AUTORES: Cada autor contribuiu individual e significativamente para o desenvolvimento do manuscrito. NVS (orcid.org/0000-0001-55284264) e PRPS (0000-0002-9460-8847) foram os principais contribuintes intelectuais do estudo e da redação do manuscrito. FPM (orcid.org/0000-0002-5303-1068), VLA (orcid.org/0000-0002-8981-3854), BLSB (orcid.org/0000-0003-3821-2327) e LHPV (orcid.org/0000-0001-6981-756X), participaram ativamente da coleta de dados, análise estatística, discussão dos resultados, revisão e aprovação da versão final do trabalho. *Número ORCID (Open Researcher and Contributor ID).

\section{REFERÊNCIAS}

1. Griffin LY, Agel J, Albohm MJ, Arendt EA, Dick RW, Garrett WE, et al. Noncontact anterior cruciate ligament injuries: risk factors and prevention strategies. J Am Acad Orthop Surg. 2000;8(3):141-50.

2. Micheo W, Hernández L, Seda C. Evaluation, management, rehabilitation, and prevention of anterior cruciate ligament injury: current concepts. PMR. 2010;2(10):935-44.

3. Hewett TE, Myer GD, Ford KR, Paterno MV, Quatman CE. The 2012 ABJS Nicolas Andry Award: The sequence of prevention: a systematic approach to prevent anterior cruciate ligament injury. Clin Orthop Relat Res. 2012;470(10):2930-40.

4. Wilson NA, Press JM, Koh JL, Hendrix RW, Zhang LQ. In vivo noninvasive evaluation of abnormal patellar tracking during squatting in patients with patellofemoral pain. J Bone Joint Surg Am. 2009;91(3):558-66.

5. Anderson AF, Dome DC, Gautam S, Awh MH, Rennirt GW. Correlation of anthropometric measurements, strength, anterior cruciate ligament size, and intercondylar notch characteristics to sex differences in anterior cruciate ligament tear rates. Am J Sports Med. 2001;29(1):58-66.

6. Hewett TE, Myer GD, Ford KR, Heidt RS Jr, Colosimo AJ, McLean SG, et al. Biomechanical measures of neuromuscular control and valgus loading of the knee predict anterior cruciate ligament injury risk in female athletes: a prospective study. Am J Sports Med. 2005;33(4):492-501.

7. Hewett TE, Paterno MV, Myer GD. Strategies for enhancing proprioception and neuromuscular control of the knee. Clin Orthop Relat Res. 2002;(402):76-94.

8. McLean SG, Lipfert SW, van den Bogert AJ. Effect of gender and defensive opponent on the biomechanics of sidestep cutting. Med Sci Sports Exerc. 2004;36(6):1008-16.

9. Slauterbeck JR, Hardy DM. Sex hormones and knee ligament injuries in female athletes. Am J Med Sci. 2001;322(4):196-9.

10. Cesar GM, Pereira VS, Santiago PR, Benze BG, da Costa PH, Amorim CF, et al. Variations in dynamic knee valgus and gluteus medius onset timing in non-athletic females related to hormonal changes during the menstrual cycle. Knee. 2011;18(4):224-30.

11. Lohmander LS, Ostenberg A, Englund M, Roos H. High prevalence of knee osteoarthritis, pain, and functional limitations in female soccer players twelve years after anterior cruciate ligament injury. Arthritis Rheum. 2004;50(10):3145-52.

12. Risberg MA, Mørk M, Jenssen HK, Holm I. Design and implementation of a neuromuscular training program following anterior cruciate ligament reconstruction. J Orthop Sports Phys Ther. 2001;31(11):620-31.

13. Swanik CB, Lephart SM, Giannantonio FP, Fu FH. Reestablishing proprioception and neuromuscular control in the ACL-injured athlete. J Sport Rehabil. 1997;6(2):182-206.

14. Russell KA, Palmieri RM, Zinder SM, Ingersoll CD. Sex differences in valgus knee angle during a singleleg drop jump. J Athl Train. 2006:41(2):166-71.

15. Malinzak RA, Colby SM, Kirkendall DT, Yu B, Garrett WE. A comparison of knee joint motion patterns between men and women in selected athletic tasks. Clin Biomech (Bristol, Avon). 2001;16(5):438-45.

16. Lephart SM, Ferris CM, Riemann BL, Myers JB, Fu FH. Gender differences in strength and lower extremity kinematics during landing. Clin Orthop Relat Res. 2002;(401):162-9.

17. Cappozzo A, Catani F, Croce UD, Leardini A. Position and orientation in space of bones during movement: anatomical frame definition and determination. Clin Biomech (Bristol, Avon). 1995 ;10(4):171-178

18. Myer GD, Ford KR, McLean SG, Hewett TE. The effects of plyometric versus dynamic stabilization and balance training on lower extremity biomechanics. Am J Sports Med. 2006;34(3):445-55.

19. Grood ES, Suntay WJ. A joint coordinate system for the clinical description of three-dimensional motions: application to the knee. J Biomech Eng. 1983;105(2):136-44.

20. Santiago PRP. Rotações tridimensionais em biomecânica via quatérnions: aplicação na análise dos movimentos esportivos [tese]. Rio Claro, SP: Instituto de Biociências do Campus de Rio Claro, Universidade Estadual Paulista "Júlio de Mesquita Filho"; 2009

21. Spanó NV, Valenti EE, Moraes R, Cunha SA, Santiago PRP. The effect of neuromuscular training on the knee angular velocity: description by quaternions representation. In: XXIV Congress of the International Society of Biomechanics, 04 de agosto a 09 de agosto, 2013, Natal, RN, Brazil,

22. Koga H, Nakamae A, Shima Y, I wasa J, Myklebust G, Engebretsen L, et al. Mechanisms for noncontact anterior cruciate ligament injuries: knee joint kinematics in 10 injury situations from female team handball andbasketball. Am J Sports Med. 2010;38(11):2218-25.

23. Blackburn JT, Padua DA. Influence of trunk flexion on hip and knee joint kinematics during a controlled drop landing. Clin Biomech (Bristol, Avon). 2008;23(3):313-9.

24. Yeow $C H$, Lee PV, Goh JC. Regression relationships of landing height with ground reaction forces, knee flexion angles, angular velocities and joint powers during double-leg landing. Knee. 2009;16(5):381-6.

25. Pollard CD, Sigward SM, Powers CM. Limited hip and knee flexion during landing is associated with increased frontal plane knee motion and moments. Clin Biomech (Bristol, Avon). 2010;25(2):142-6.

26. Myer GD, Chu DA, Brent JL, Hewett TE. Trunk and hip control neuromuscular training for the prevention of knee joint injury. Clin Sports Med. 2008;27(3):425-48.

27. Baldon RM, Lobato DF, Carvalho LP, Wun PY, Santiago PR, Serrão FV. Effect of functional stabilization training on lower limb biomechanics in women. Med Sci Sports Exerc. 2012;44(1):135-45.

28. Stearns KM, Keim RG, Powers CM. Influence of relative hip and knee extensor muscle strength on landing biomechanics. Med Sci Sports Exerc. 2013;45(5):935-41.

29. Chappell JD, Limpisvasti O. Effect of a neuromuscular training program on the kinetics and kinematics of jumping tasks. Am J Sports Med. 2008;36(6):1081-6.

30. Myer GD, Paterno MV, Ford KR, Hewett TE. Neuromuscular training techniques to target deficits before return to sport after anterior cruciate ligament reconstruction. J Strength Cond Res. 2008;22(3):987-1014. 